\title{
Probing the Bottom End of the IMF in Orion with Gemini
}

\author{
Phil W. Lucas \\ Dept of Physical Sciences, University of Hertfordshire, College Lane, \\ Hatfield AL10 9AB, UK \\ Patrick F. Roche and Fiona C. Riddick \\ Astrophysics Dept., University of Oxford, 1 Keble Road, Oxford OX1 \\ $3 R H$
}

\begin{abstract}
We present very deep JHK imaging of the Trapezium Cluster obtained with Gemini South/Flamingos and Gemini North/Hōkūpa'a. These images probe the IMF down to $\sim 2 M_{J u p}$ in a total area of $\sim 8 \operatorname{arcmin}^{2}$. Several very faint new planetary mass candidates are detected and sources previously detected with UKIRT are verified. Photometry of 124 point sources in this field produces a Luminosity Function which drops to zero at $K=18.75$. Allowing for modest extinction this corresponds to a possible turn-down in the IMF near 5 Mjup. A minority of $\mathrm{PMOs}$ exhibit large $\mathrm{K}$ band excesses attributed to hot dust, confirming their extreme youth. Some of the faintest sources are associated with short trails of light of uncertain nature. These may provide a clue to the origin of PMOs, perhaps marking evacuated paths cleared by rapidly moving objects.
\end{abstract}

\section{Introduction}

Large populations of substellar objects have been detected in several very young clusters in recent years, with the mass functions apparently extending to below the deuterium burning (planetary) threshold at 0.012-0.013 $\mathrm{M}_{\odot}$ in many cases, e.g.,. Lucas \& Roche 2000; Zapatero-Osorio et al. 2000). Several of these results have now been spectroscopically confirmed. The masses are derived from theoretical isochrones based on spherical collapse models with no account of the star formation environment so the uncertainty is substantial at present, though difficult to quantify.

The detection of objects with inferred masses below the deuterium burning limit (PMOs) may challenge our present understanding of star formation. Simple arguments based on gravitational collapse of molecular cloud cores suggest that the minimum Jeans mass is near $0.01 \mathrm{M}_{\odot}$ (e.g.,. Silk 1977), though the SPH simulations of Bate, Bonnell \& Bromm (2002) use an equation of state which leads to a slightly lower Jeans mass. If there is a Jeans mass problem, solutions include: (1) photoevaporation of isolated protostars and proto-brown dwarfs; (2) complicated magnetic field effects which reduce the Jeans mass by lowering the temperature of the protostellar matter (Boss 2001); (3) 3-body encounters 
between stars and protostars which eject the protostar from its cloud core before the accretion process is complete (Reipurth \& Clarke 2001). Ejection of orbiting extrasolar 'superplanets' is also conceivable (Smith \& Bonnell 2001) following recent radial velocity detections of several objects with $m . \sin (i)>8 M_{J u p}$ (e.g.,. Udry et al. 2002). Since the Initial Mass Function (IMF) is a key test of any star formation theory it is very important to determine whether it has a lower limit. Here we present preliminary observations to address this question.

\section{Observations}

A region of the Trapezium Cluster 3 arcmin due south of $\theta_{1}$ Ori $\mathrm{C}$ was imaged with the 8-m Gemini South telescope in October 2001. The near infrared camera Flamingos (built at the University of Florida) was used to image in the $\mathrm{J}, \mathrm{H}$ and $\mathrm{K}$ bands, with approximate total exposure times of 2 hours, 1 hour and 1 hour respectively, coadded over several nights. Image quality was good (0.4-0.45 arcsec FWHM in the coadded data). Flamingos has a $2048 \times 2048$ HAWAII 2 array with a pixel scale of 0.078 arcsec/pixel, giving a 160 arcsecond field of view. A single field was imaged with small offset jitter pattern.

This field was selected since it lies on top of the OMC-1 molecular ridge, which was expected to heavily redden or completely obscure background stars across most of the field. The field contains some moderately bright nebulosity in the northern half, associated with the Orion Bar. Sensitivity is therefore non-uniform, being better in the southern half of the field. Similar additional observations to the west of the OMC-1 ridge are planned for winter 2002/3.

The Gemini North telescope was also used to image five 20 arcsec fields with the Hōkūpa'a adaptive optics system in December 2001, in the $\mathrm{J}$ and $\mathrm{H}$ bands only. The detector was a HAWAII 1 array in the infrared camera QUIRC. The image quality was 0.18 arcsec FWHM and the integration time was 20 minutes per filter for each field. Four fields were located 4.5 arcmin southwest of $\theta_{1}$ Ori C, in a small subcluster. One field was located close to the core of the Trapezium cluster, where stellar density is highest. This paper deals mainly with the Gemini South/Flamingos data.

\section{Results and Discussion}

\subsection{Photometry}

124 point sources were detected in the Flamingos field at $\mathrm{K}$ band. Nearly all were detected at $\mathrm{J}$ and $\mathrm{H}$ also: only 9 sources were undetected at $\mathrm{J}$ and only 2 were undetected at $\mathrm{H}$. Fluxes for each source were measured with the APPHOT package in IRAF, using manually selected sky annuli for correct subtraction of the spatially variable nebulous background. The results presented here are based on a preliminary photometric calibration, derived from measurements of 9 bright unsaturated 2MASS sources in the field. The $\mathrm{K}$ band luminosity function (KLF) is shown in Figure 1(a). A two colour diagram is shown in Figure 1(b).

The KLF shows a cut-off beyond the bin at $K=18.5$. A single possible detection at $K=19.4$ is probably a filament of nebulosity: it appears point sourcelike in the $\mathrm{H}$ band but is highly elongated in the $\mathrm{J}$ and $\mathrm{K}$ bands. About 7 sources 

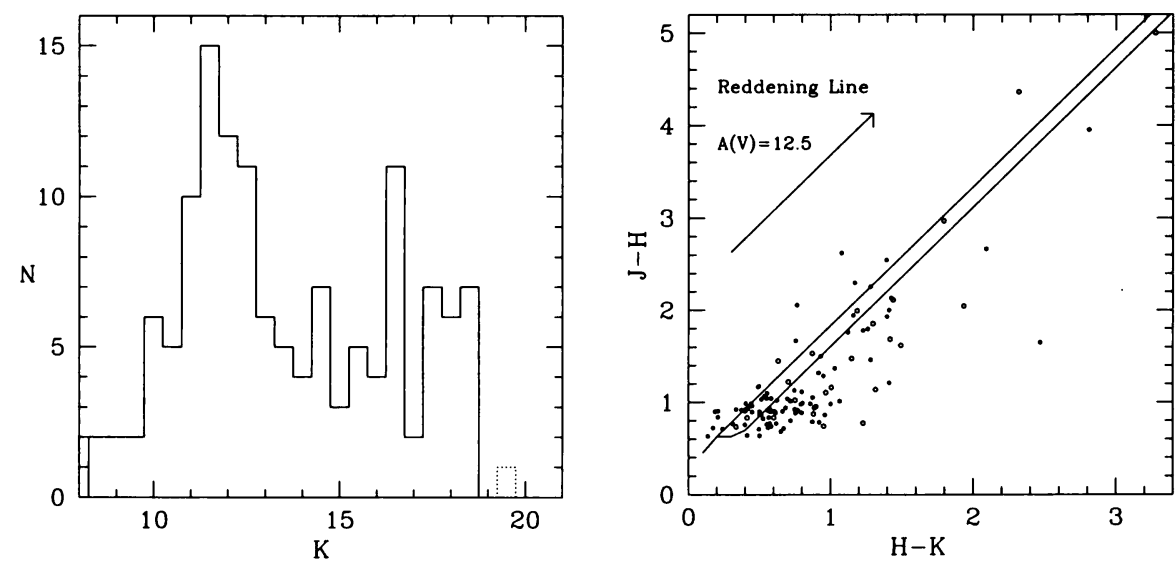

Figure 1. Left: (a) - the KLF, 90\% complete to $\mathrm{K}=19.25,50 \%$ complete to $K=19.75$. Right: (b) - two colour diagram, with reddening lines from an approximate main sequence.

per bin are detected in the 3 bins up to the cut-off. The sensitivity limit was determined by generated artificial stars in IRAF. This indicates that the next bin at $K=19.0$ is at least $90 \%$ complete. The following bin at $K=19.5$ is about $50 \%$ complete: it is complete in the southern half of the image (where the nebulosity is fainter) but in the northern half the artificial stars were not convincingly distinguished from nebulosity. Assuming the KLF is sampled from an underlying function determined by star formation physics with shot-noise statistics, the absence of sources in the $\mathrm{K}=19.0$ bin implies a drop in the luminosity function with $98 \%$ confidence. The apparent cut-off occurs at a mass $M \sim 5 M_{J u p}$, using the Burrows et al. (1997) isochrones. The Baraffe et al. (1998) isochrones appear to imply a slightly lower mass.

The drop in the KLF may be taken as evidence for a turn down in the IMF, supporting earlier indications (Lucas \& Roche 2000). The Hōkūpa'a data show a similar absence of very faint sources in fields containing 25 point sources. However, the sample is far too small to establish whether this is a cut-off. The change in the LF might also be due to an unexpected change in the slope of the mass-luminosity relation, or a quirk of the physics in this particular field. The projected field location is behind the ionisation front marked by the Orion Bar, so there may be some shielding from photoevaporation.

The two-colour diagram (Figure 1(b)) indicates that some sources lie well to the right of the reddening line, implying that a minority of sources have large $\mathrm{K}$ band excesses due to hot dust. This appears to be true of very low mass sources (open circles) just as often as stars (solid circles), suggesting that the PMO candidates are very young $(<3 \mathrm{Myr})$, apparently confirming and extending the result of Muench et al. (2001) and McCaughrean et al. (1995). However, this is only a preliminary result, since it is sensitive to the photometric calibration and to $\mathrm{T}$ Tauri-like source variability. 


\subsection{Resolved Circumstellar Matter}

A minority of sources display short trails in the Flamingos JHK data, typically a few arcsec in length. The interpretation of these features is unclear: there are also filaments of nebulosity which are not associated with a point source, some of which may relate to the Orion V-shaped molecular 'bullets' which pervade the cluster e.g.,. Lee \& Burton (2000). Nevertheless, we argue that some of the PMOs are physically associated with these trails. The trails are mostly associated with faint sources (PMO candidates) and generally extend northward from the continuum source in the general direction of the O-type Trapezium stars. This orientation indicates that they are not proplyd structures shaped by photoevaporation. One possibility is that they are illuminated tunnels cleared in the neutral cluster medium by the passage of rapidly moving bodies. This has been previously suggested as the explanation of filamentary 'umbilical cords' in Serpens (Eiroa \& Casali 1992). However, at least 1 source with a trail appears to be embedded within a dense structure, judging by the high extinction.

To establish the nature of the trails, it would be useful to obtain proper motion data for the PMOs, to see whether the trails can be associated with source motion and whether the PMOs have unusually high space velocities. This might be expected by an equipartition of energy argument if they were ejected from multiple systems. Narrow band imaging might also be used to asses whether the trails have a photoionised skin like proplyds.

\section{References}

Baraffe I., Chabrier G., Allard F., \& Hauschildt P.H. 1998, A\&A, 337,403

Bate M.R., Bonnell I.A. \& Bromm V. 2002, MNRAS, 332, L65

Boss A. 2001, ApJ, 551, L167

Burrows A., Marley M., Hubbard W.B., Lunine J.I., Guillot T., Saumon D., Freedman R., Sudarsky D., \& Sharp C. 1997, ApJ, 491,856

Eiroa C. \& Casali M.M. 1992, A\&A, 262, 468

Lee J-K. \& Burton M.G. 2000, MNRAS, 315, 11

Lucas P.W. \& Roche P.F. 2000, MNRAS, 314, 858

McCaughrean M., Zinnecker H., Rayner J., \& Stauffer J. 1995, in "The Bottom of the Main Sequence and Beyond", ed. C. Tinney, p209, (Berlin: Springer)

Muench A.A., Alves J., Lada C.J., Lada E.A. 2001, ApJ, 558, L51

Reipurth B. \& Clarke C. 2001, AJ, 122, 432

Silk J. 1977, ApJ, 214, 152

Smith K. \& Bonnell I. 2001, MNRAS, 322, L1

Udry S., Mayor M., Naef D., Pepe F., Queloz D., Santos N.C., Burnet M. 2002 A\&A, 390, 267

Zapatero Osorio M.R., Béjar V.J.S., Martín E.L., Rebolo R., Barrado y Navascués D., Bailer-Jones C., \& Mundt R. 2000, Science 290, 103 